\title{
Change-point estimation for repairable systems combining bootstrap control charts and clustering analysis: Performance analysis and a case study
}

\author{
Yang, Z.J. ${ }^{a}$, Du, X.J. ${ }^{a}$, Chen, F. ${ }^{\text {a, }}{ }^{*}$, Chen, C.H. ${ }^{\text {a, }}{ }^{,}$, Tian, H.L. ${ }^{a}$, He, J.L. ${ }^{a}$ \\ ${ }^{a}$ School of Mechanical Science and Engineering, Jilin University, Changchun, P.R. China
}

\section{A B S T R A C T}

Complex repairable systems with bathtub-shaped failure intensity will normally go through three periods in the lifecycle, which requires maintenance policies and management decisions accordingly. Therefore, the accurate estimation of change points of different periods has great significance. This paper addresses the challenge of change-point estimation in failure processes for repairable systems, especially for sustained and gradual processes of change. The paper proposes a sectional model composed of two non-homogeneous Poisson processes (NHPPs) to describe the bathtub-shaped failure intensity. In order to obtain the accurate change-point estimator, a novel hybrid method is developed combining bootstrap control charts with the sequential clustering approach. Through Monte Carlo simulations, the proposed change-point estimation method is compared with two powerful estimation procedures in various conditions. The results suggest that the proposed method performs effective and satisfactory for failure processes with no limits of distributions, changing ranges and sampling schemes. It especially provides higher precision and lower uncertainty in detecting small shifts of change. Finally, a case study analysing real failure data from a heavy-duty CNC machine tool is presented. The parameters of the proposed NHPP model are estimated. The change point of the early failure period and the random failure period is also calculated. These findings can contribute to determining the burn-in time in order to improve the reliability of the machine tool.
\end{abstract}

\section{ARTICLE INFO}

\section{Keywords:}

Change-point estimation;

CNC machine tools;

Non-homogeneous Poisson process

(NHPP);

Statistical process control (SPC)

Bathtub-shape behaviour;

Clustering

*Corresponding author:

chenfeicn@jlu.edu.cn

(Chen, F.)

cchchina@foxmail.com

(Chen, C.H.)

Article history:

Received 17 September 2017

Revised 27 August 2018

Accepted 29 August 2018

\section{References}

[1] Amiri, A., Allahyari, S. (2012). Change point estimation methods for control chart postsignal diagnostics: A literature review, Quality and Reliability Engineering International, Vol. 28, No. 7, 673-685, doi: 10.1002/qre.1266.

[2] Pignatiello Jr, J.J., Samuel, T.R. (2001). Estimation of the change point of a normal process mean in SPC applications, Journal of Quality Technology, Vol. 33, No. 1, 82-95, doi: 10.1080/00224065.2001.11980049.

[3] Perry, M.B., Pignatiello Jr, J.J. (2006). Estimation of the change point of a normal process mean with a linear trend disturbance in SPC, Quality Technology \& Quantitative Management, Vol. 3, No. 3, 325-334, doi: 10.1080/168437 $\underline{03.2006 .11673118 .}$

[4] Perry, M.B., Pignatiello Jr, J.J., Simpson, J.R. (2007). Change point estimation for monotonically changing Poisson rates in SPC, International Journal of Production Research, Vol. 45, No. 8, 1791-1813, doi: 10.1080/002075406 $\underline{00622449}$.

[5] Hawkins, D.M., Zamba, K.D. (2005). Statistical process control for shifts in mean or variance using a change point formulation, Technometrics, Vol. 47, No. 2, 164-173, doi: 10.1198/004017004000000644.

[6] Zamba, K.D., Hawkins, D.M. (2006). A multivariate change-point model for statistical process control, Technometrics, Vol. 48, No. 4, 539-549, doi: 10.1198/004017006000000291.

[7] Holland, M.D., Hawkins, D.M. (2014). A control chart based on a nonparametric multivariate change-point model, Journal of Quality Technology, Vol. 46, No. 1, 63-77, doi: 10.1080/00224065.2014.11917954. 
[8] Weese, M., Martinez, W., Megahed, F.M., Jones-Farmer, L.A. (2016). Statistical learning methods applied to process monitoring: An overview and perspective, Journal of Quality Technology, Vol. 48, No. 1, 4-24, doi: 10.1080/ 00224065.2016 .11918148$.

[9] Amiri, A., Niaki, S.T.A., Moghadam, A.T. (2015). A probabilistic artificial neural network-based procedure for variance change point estimation, Soft Computing, Vol. 19, No. 3, 691-700, doi: 10.1007/s00500-014-1293-x.

[10] Ahmadzadeh, F. (2009). Change point detection with multivariate control charts by artificial neural network, The International Journal of Advanced Manufacturing Technology, Vol. 97, No. 9-12, 3179-3190, doi: 10.1007/s001 70-009-2193-6.

[11] Ahmadzadeh, F., Lundberg, J., Strömberg, T. (2013). Multivariate process parameter change identification by neural network, The International Journal of Advanced Manufacturing Technology, Vol. 69, No. 9-12, 2261-2268, doi: $10.1007 / \mathrm{s} 00170-013-5200-\mathrm{x}$.

[12] Maleki, M.R., Amiri, A., Mousavi, S.M. (2015). Step change point estimation in the multivariate-attribute process variability using artificial neural networks and maximum likelihood estimation, Journal of Industrial Engineering International, Vol. 11, No. 4, 505-515, doi: 10.1007/s40092-015-0117-7.

[13] Ghiasabadi, A., Noorossana, R., Saghaei, A. (2013). Identifying change point of a non-random pattern on $\bar{X}$ control chart using artificial neural networks, The International Journal of Advanced Manufacturing Technology, Vol. 67, No. 5-8, 1623-1630, doi: 10.1007/s00170-012-4595-0.

[14] Zarandi, M.H.F., Alaeddini, A. (2010). A general fuzzy-statistical clustering approach for estimating the time of change in variable sampling control charts, Information Sciences, Vol. 180, No. 16, 3033-3044, doi: 10.1016/i.ins.2010.04.017.

[15] Alaeddini, A., Ghazanfari, M., Nayeri, M.A. (2009). A hybrid fuzzy-statistical clustering approach for estimating the time of changes in fixed and variable sampling control charts, Information Sciences, Vol. 179, No. 11, 17691784, doi: 10.1016/i.ins.2009.01.019.

[16] Ghazanfari, M., Alaeddini, A., Niaki, S.T.A., Aryanezhad, M.-B. (2008). A clustering approach to identify the time of a step change in Shewhart control charts, Quality and Reliability Engineering International, Vol. 24, No. 7, 765778, doi: 10.1002 /qre.925.

[17] Kazemi, M.S., Bazargan, H., Yaghoobi, M.A. (2014). Estimating the drift time for processes subject to linear trend disturbance using fuzzy statistical clustering, International Journal of Production Research, Vol. 52, No. 11, 33173330, doi: $10.1080 / 00207543.2013 .872312$.

[18] Zarandi, M.H.F., Najafi, S. (2015). A type-2 fuzzy-statistical clustering approach for estimating the multiple change points in a process mean with monotonic change, The International Journal of Advanced Manufacturing Technology, Vol. 77, No. 9-12, 1751-1765, doi: 10.1007/s00170-014-6570-4.

[19] Kazemi, M.S., Kazemi, K., Yaghoobi, M.A., Bazargan, H. (2016). A hybrid method for estimating the process change point using support vector machine and fuzzy statistical clustering, Applied Soft Computing, Vol. 40, 507-516, doi: 10.1016/i.asoc.2015.11.021.

[20] He, S., Jiang, W., Deng, H. (2018). A distance-based control chart for monitoring multivariate processes using support vector machines, Annals of Operations Research, Vol. 263, No. 1-2, 191-207, doi: 10.1007/s10479-0162186-4.

[21] Ebeling, C.E. (2004). An introduction to reliability and maintainability engineering, Tata McGraw-Hill Education, New Delhi, India.

[22] Jiang, R. (2015). Introduction to quality and reliability engineering, Springer, New York, USA, doi: 10.1007/978-3662-47215-6.

[23] Jensen, W.A., Jones-Farmer, L.A., Champ, C.W., Woodall, W.H. (2006). Effects of parameter estimation on control chart properties: A literature review, Journal of Quality Technology, Vol. 38, No. 4, 349-364, doi: 10.1080/002240 65.2006.11918623.

[24] Zhang, M., Megahed, F.M., Woodall, W.H. (2014). Exponential CUSUM charts with estimated control limits, Quality and Reliability Engineering International, Vol. 30, No. 2, 275-286, doi: 10.1002/qre.1495.

[25] Nichols, M.D., Padgett, W.J. (2006). A bootstrap control chart for Weibull percentiles, Quality and Reliability Engineering International, Vol. 22, No. 2, 141-151, doi: 10.1002/qre.691.

[26] Zhang, Y., Qiu, J., Liu, G., Zhao, Z. (2015). Fault sample generation for virtual testability demonstration test subject to minimal maintenance and scheduled replacement, Mathematical Problems in Engineering, Vol. 2015, Article ID 645047, doi: 10.1155/2015/645047.

[27] Samuel, T.R., Pignatiello Jr., J.J., Calvin, J.A. (1998). Identifying the time of a step change with $\bar{X}$ control charts, Quality Engineering, Vol. 10, No. 3, 521-527, doi: 10.1080/08982119808919166. 


\section{APEM}

Advances in Production Engineering \& Management Letnik 13 | Številka 3 | September 2018 | Strani 307-320 https://doi.org/10.14743/apem2018.3.292
ISSN 1854-6250

Spletna stran: apem-journal.org Izvirni znanstveni članek

\title{
Ocena točke spremembe pri popravljivih sistemih z združitvijo bootstrap nadzornih grafov in analizo združevanja gruč: Analiza uspešnosti in študija primera
}

\author{
Yang, Z.J. ${ }^{a}$, Du, X.J. ${ }^{a}$, Chen, F. ${ }^{a}{ }^{,}$, Chen, C.H. ${ }^{a},{ }^{,}$, Tian, H.L. ${ }^{a}$, He, J.L. ${ }^{a}$ \\ ${ }^{\mathrm{a}}$ School of Mechanical Science and Engineering, Jilin University, Changchun, P.R. China
}

\section{POVZETEK}

Kompleksni popravljivi sistemi z intenzivnostjo okvare v obliki kopalne kadi, $\mathrm{v}$ času življenjskega cikla preidejo tri obdobja, kar zahteva ustrezno vzdrževalno politiko in odločitve o upravljanju. Zato je zelo pomembna natančna ocena točke spremembe med različnimi obdobji. V prispevku je predstavljeno ocenjevanje točke spremembe pri okvarah na popravljivih sistemih; poudarek je na trajnih in postopnih procesnih spremembah. V prispevku je predlagan model, sestavljen iz dveh nehomogenih Poissonovih procesov (NHPPs), ki opisujeta intenziteto okvare v obliki kopalne kadi. Za pridobitev natančne ocene točke spremembe je bila razvita nova hibridna metoda, ki združuje bootstrap nadzorne grafe in postopek zaporednega združevanja gruč. S simulacijami Monte Carlo smo predlagano metodo ocenjevanja točke spremembe primerjali z dvema ocenjevalnima postopkoma pri različnih pogojih. Rezultati so pokazali, da predlagana metoda deluje učinkovito in zadovoljivo pri procesih okvare brez omejitev distribucije, spreminjanja razporedov in shem vzorčenja. Zagotavlja večjo natančnost in manjšo negotovost, zlasti pri odkrivanju majhnih premikov sprememb. Na koncu je predstavljena še študija primera, ki analizira dejanske podatke o napakah industrijskega CNC stroja. Ocenjeni so bili parametri predlaganega modela NHPP ter točka spremembe med okvaro v zgodnjem obdobju in naključnim izpadom. Te ugotovitve lahko prispevajo $\mathrm{k}$ napovedi trenutka izpada in povečanju zanesljivost strojev.
\end{abstract}

\section{PODATKI O ČLANKU}

Ključne besede:

Ocena točke spremembe;

CNC stroji;

Nehomogeni Poissonovi procesi

(NHPP);

Statistični nadzor procesov;

Odvisnosti v obliki kopalne kadi

Združevanje gruč

*Kontaktna oseba:

chenfeicn@jlu.edu.cn

(Chen, F.)

cchchina@foxmail.com

(Chen, C.H.)

Zgodovina članka:

Prejet 17. septembra 2017

Popravljen 27. avgusta 2018

Sprejet 24. avgusta 2018 